\title{
Nématodes Spirura parasites des Tupaia et du Nycticèbe en Malaisie
}

\author{
par J.-Cl. QUENTIN * et M. KRISHNASAMY ** \\ * Laboratoire de Zoologie (Vers), associé au C.N.R.S., \\ Muséum national d'Histoire naturelle, 43, rue Cuvier, F 75231 Paris Cedex 05 \\ ** Division of Medical Ecology (Mr. Lim Boo Liat), \\ Institute for Medical Research, Kuala Lumpur, Malaysia.
}

\section{Résumé.}

Etude morphologique de deux Spirura parasites de la paroi œsophagienne et stomacale des Tupaia et du Nycticèbe en Malaisie.

- Spirura malayensis n. sp. est récolté à la fois chez les Tupaia de la province de Selangor (Malaisie occidentale) et chez le Nycticebus coucang à Bornéo. Ses caractères très primitifs le rapprochent de l'espèce $S$. diplocyphos Chabaud, Brygoo et Petter, 1965, parasite de Lémurien malgache.

Son développement larvaire est obtenu expérimentalement chez Blatella germanica.

- Spirura aurangabadensis (Ali et Lovekar, 1966), décrit chez un Microchiroptère aux Indes, est récolté chez un Nycticebus coucang et chez un Tupaia glis de Malaisie occidentale.

- La répartition des espèces et l'étude comparée des structures céphaliques larvaires et adultes, montrent que le genre Spirura s'est fondamentalement diversifié dans l'Ancien Monde chez des hôtes très primitifs selon deux lignées évolutives principales.

\section{Summary.}

Nematodes of the genus Spirura, parasites of Tupaia and Nycticebus in Malaysia.

Morphological study of two Spirura parasites of the oesophageal and the gastric wall of Tupaia and Nycticebus in Malaysia. 
- Spirura malayensis $\mathrm{n}$. sp. is found both in Tupaia in the District of Selangor (West Malaysia) and in Nycticebus coucang in Borneo. Its very primitive characteristics relate it to $S$. diplocyphos Chabaud, Brygoo and Petter, 1965, parasite of lemurs from Madagascar.

Its larval development was obtained experimentally in Blatella germanica.

- Spirura aurangabadensis (Ali and Lovekar, 1966) described from a microchiroptera in India is found in west Malaysia in a Nycticebus coucang, and in a Tupaia glis.

- The distribution of the different species and the comparative study of the larval and adult cephalic structures show that the genus Spirura arose and became diversified in the old world in very primitive hosts according to two main evolutive lines.

\section{Introduction}

Trois Spirurides sont parasites de la paroi œsophagienne et stomacale des Tupaia et du Nycticebus coucang en Malaisie.

L'un d'entre eux, Gongylonema neoplasticum Fibiger et Ditlevsen, 1914 (fig. 1), est récolté dans la paroi œsophagienne d'un Tupaia glis (Diard) à la station biologique de Sepilok, Sandakan, Province de Sabah. Il s'agit d'un parasite fréquemment récolté chez le Rattus, qui est cosmopolite et qui peut évoluer chez des hôtes très différents. Il n'est donc pas étonnant de le récolter à Bornéo chez le Tupaia.

Les deux autres Spirurides appartiennent au genre Spirura et diffèrent profondément par leur morphologie.

- Spirura malayensis n. sp. présente des caractères très primitifs qui le rapprochent des Spirura de Lémuriens malgaches et africains. Fréquent chez les Tupaia de la Province de Selangor (Malaisie occidentale), ce Spirura est identifié également chez le Nycticebus coucang à Bornéo.

- Spirura aurangabadensis (Ali et Lovekar, 1966) a par contre une morphologie très différenciée. Décrit initialement chez un Microchiroptère Taphozous kacchensis aux Indes, ce parasite est récolté en Malaisie occidentale chez un Nycticebus coucang et chez un Tupaia glis.

Nous précisons la morphologie de ces deux dernières espèces, puis nous tentons de situer leur place zoologique respective dans l'évolution du genre Spirura.

\section{I. - Morphologie des espèces Spirura malayensis n. sp. et Spirura aurangabadensis (Ali et Lovekar, 1966)}

\section{Spirura malayensis n. sp.}

Hôte : Tupaia glis (Diard).

Localité : W. Malaysia, Selangor Gombak, Ulu Gombak, Forest Reserve.

Dates de récoltes et $\mathrm{n}^{\circ}$ d'enregistrement : 24-12-73, M.N.H.N. $\mathrm{n}^{\circ}$ KL 122 (dont $1 \delta^{*}$ holotype et $1 q$ allotype), KL $123 ; 25-12-73, \mathrm{n}^{\circ} \mathrm{KL} 124 ; 2-1-74, \mathrm{n}^{\circ} \mathrm{KL} 161$; 


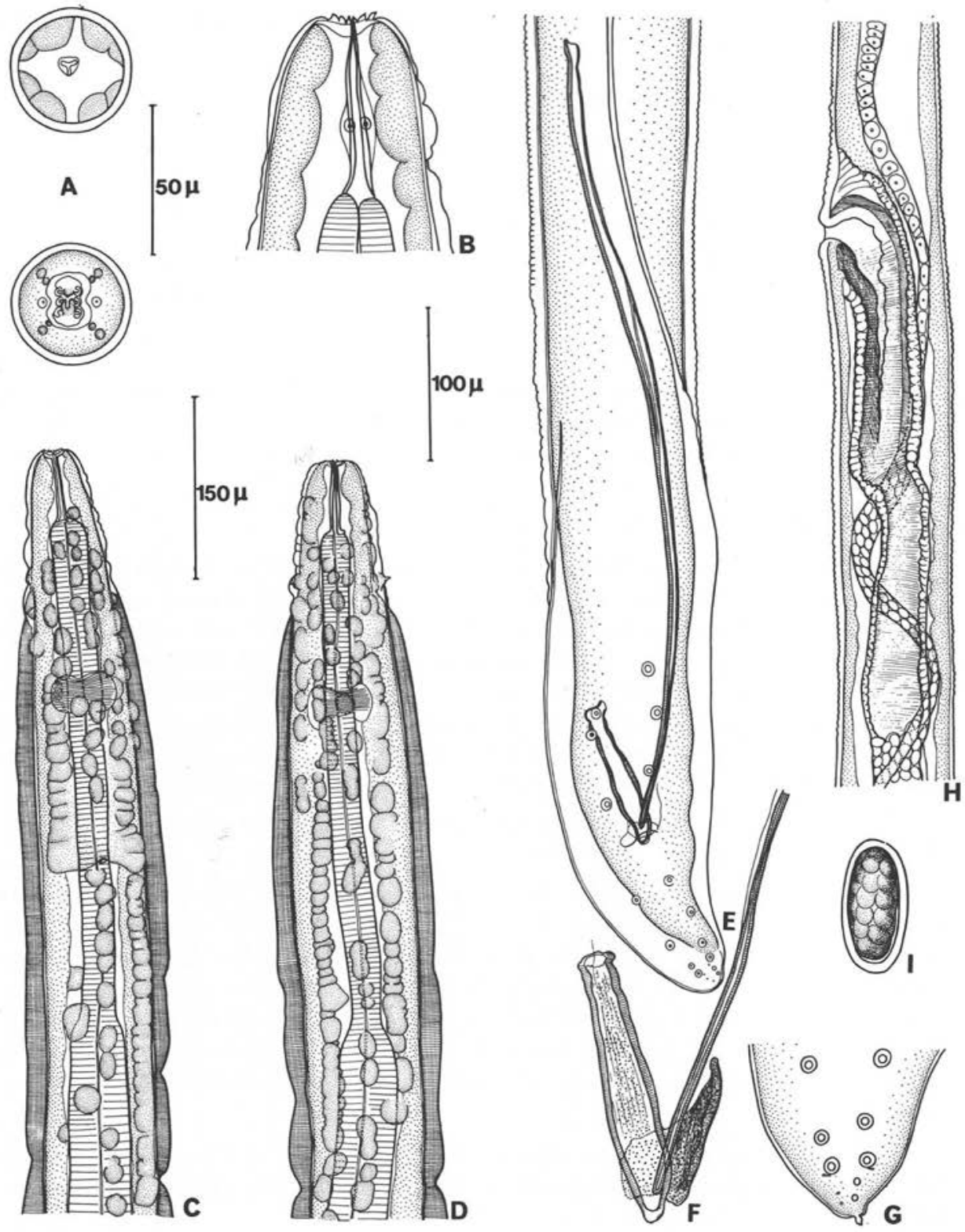

FIG. 1. - Gongylonema neoplasticum Fibiger Ditlevsen, 1914. A : tête représentée en vue apicale et en coupe optique au niveau du pharynx; B : tête en vue ventrale ; C et D : détail des bosses cuticulaires sur les faces ventrale et dorsale du corps de la $\uparrow$; E : bourse caudale du $\delta$, vue ventrale; F : extrémité des spicules (longueurs $98 \mu$ et $530 \mu$ ) et détail du gubernaculum (longueur $111 \mu$ ) ; H : ovéjecteur; G : pointe caudale du mâle; I : œuf. A, B, F, G, I: éch. $50 \mu ;$ C, D, H: éch. $150 \mu$; E: éch. $100 \mu$. 
$3-1-74, \mathrm{n}^{\circ}$ KL $162 ; 8-2-74, \mathrm{n}^{\circ}$ KL $258 ; 9-2-74, \mathrm{n}^{\circ}$ KL $260 ; 11-2-74, \mathrm{n}^{\circ}$ KL 263 ; 14-2-74, $\mathrm{n}^{\circ}$ KL 266.

Hôte : Tupaia minor Günther.

Localité : idem.

Date de récolte: $25-12-73, \mathrm{n}^{\circ} \mathrm{KL} 126$.

Hôte: Nycticebus coucang (Boddaert).

Localité : Kota Kinabalu, Sabah.

Date de récolte et $\mathrm{n}^{\circ}$ d'enregistrement: 29-3-67, Division of Medical ecology $n^{\circ} 4197$.

Matériel étudié : nombreux spécimens mâles et femelles. Ce parasite paraît en effet très fréquent chez les Tupaia de Malaisie occidentale. Dix Tupaia sur quinze capturés dans la province de Selangor hébergent ce parasite.

\section{Morphologie des adultes.}

Ce Spirura a une morphologie très primitive. La tête (fig. $2 A-B-C$ ), identique dans les deux sexes, présente un cadre buccal comprimé latéralement au niveau des amphides, qui se dilate sur les faces dorsale et ventrale en une collerette aux rebords arrondis. Ce cadre porte six formations dentiformes légèrement dédoublées. Il existe quatre petites papilles labiales cachées sous le rebord de la collerette buccale et quatre grosses papilles céphaliques. Le pharynx est comprimé latéralement. Les deirides sont symétriques et très antérieures. Deux ailes latérales (fig. $2 \mathrm{E}$ ) naissent au niveau de l'anneau nerveux, et se terminent vers la fin de la région œsophagienne.

La particularité de ce Spirura réside dans la forme de la bosse cuticulaire œsophagienne ventrale qui est dédoublée.

Mâles: Il existe quatre paires de papilles et une papille impaire précloacales et six paires de papilles postcloacales (fig. $2 \mathrm{H}$ et $\mathrm{I}$ ). Ces dernières sont réparties de la façon suivante: une paire est isolée en arrière du cloaque, deux paires sont ensuite regroupées, mais sur certains spécimens la position des papilles d'une de ces deux paires peut varier, une paire est de nouveau isolée ; enfin, vers l'extrémité de la bourse caudale se trouvent deux petites paires de papilles et une paire de phasmides. Les spicules (fig. $2 J-K$ ) sont subégaux, le gauche, ailé à son extrémité distale, est plus trapu que le droit.

Femelles: L'ovéjecteur est simple (fig. 2 L). Le vagin se termine par un petit sphincter. Une trompe utérine impaire dirigée vers l'arrière le prolonge et se divise ensuite en deux utérus.

\section{Dimensions des spécimens parasites du Tupaia}

Mâle holotype: Ses dimensions sont: longueur $13750 \mu$; largeur $230 \mu$; deirides, anneau nerveux et pore excréteur situés respectivement à $160 \mu, 260 \mu$ et $330 \mu$; 

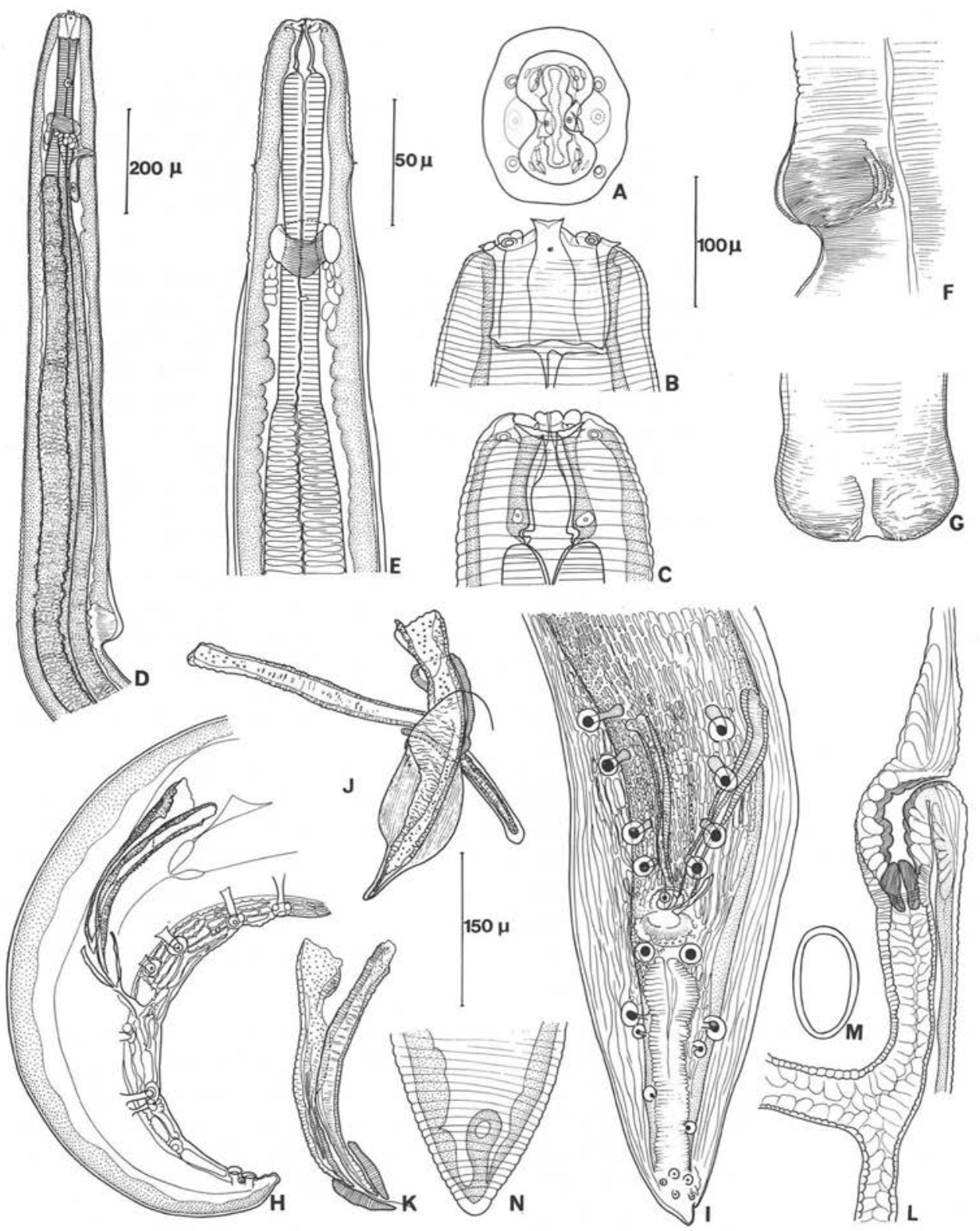

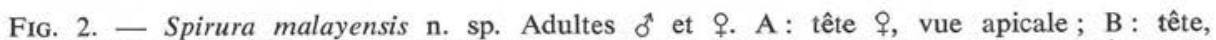
vue latérale ; C: tête, vue dorsale; D : extrémité antérieure du corps, vue ventrale montrant les deirides et les ailes latérales; $F$ et $G$ : bosses cuticulaires ventrales en vue latérale et ventrale; $\mathrm{H}$ et $\mathrm{I}$ : bourse caudale, mâle, en vue latérale et ventrale ; $\mathrm{J}$ et $\mathrm{K}$ : spicules et gubernaculum représentés en vue ventrale, les pointes des spicules étant sorties du cloaque, et en vue latérale droite; $\mathrm{L}$ : ovéjecteur; $\mathrm{M}$ : œuf; $\mathrm{N}$ : extrémité caudale femelle., A, B, C, M, N : éch. $50 \mu$; D, éch. $200 \mu$; E, H, I, L : éch. $150 \mu ;$ F, G, J, K : éch. $100 \mu$. Annales de Parasitologie humaine et comparée (Paris), t. 50, $\mathrm{n}^{\circ} 6$ 
bosse cuticulaire large de $140 \mu$, située à $1400 \mu$ de l'apex ; profondeur du pharynx $50 \mu$; longueurs respectives de l'œsophage musculaire et de l'œsophage glandulaire $320 \mu$ et $5500 \mu$; contour testiculaire remontant à $6650 \mu$ de l'apex; longueur du spicule gauche $200 \mu$; longueur du spicule droit $220 \mu$ (ces dimensions sont respectivement $188 \mu$ et $208 \mu, 200 \mu$ et $238 \mu$ sur deux autres spécimens); longueur du gubernaculum $55 \mu(50-76 \mu)$; longueur de la queue $245 \mu$.

Femelle allotype: Ses dimensions sont : longueur $13720 \mu$; largeur $300 \mu$; deirides, anneau nerveux et pore excréteur situés respectivement à $135 \mu, 220 \mu$ et $280 \mu$ de l'apex; bosse cuticulaire large de $140 \mu$, située à $1250 \mu$ de l'apex ; profondeur du pharynx $50 \mu$; longueurs respectives de l'œsophage musculaire et de l'œsophage glandulaire $270 \mu$ et $550 \mu$; vagin situé à $9800 \mu$ de l'apex ; longueur du vagin $150 \mu$; longueur de la trompe utérine impaire $175 \mu$; dimensions des œufs 45-46 $\mu \times 24-26 \mu$; longueur de la queue $225 \mu$.

\section{Dimensions des spécimens parasites du Nycticebus coucang}

Nous n'avons pas relevé de différences morphologiques au niveau des structures céphaliques et des structures génitales mâles et femelles, entre les Spirura parasites du Nycticebus coucang à Bornéo ( $\mathrm{n}^{\circ}$ d'enregistrement D.M.E. : 4197) et ceux que nous venons de décrire, parasites des Tupaia en Malaisie occidentale.

Les dimensions d'un mâle et d'une femelle de Spirura, parasites du Nycticèbe $\mathrm{n}^{\circ} 4197$, sont les suivantes :

Mâle: Longueur $18,5 \mathrm{~mm}$, largeur $400 \mu$, deirides, anneau nerveux et pore excréteur situés respectivement à $170 \mu, 300 \mu$ et $400 \mu$ de l'apex, bosse cuticulaire large de $240 \mu$, située à $1,8 \mathrm{~mm}$ de l'apex; longueurs respectives du pharynx, de l'œsophage musculaire et de l'œsophage glandulaire $60 \mu, 400 \mu$ et $7550 \mu$, contour testiculaire situé à $9 \mathrm{~mm}$ de l'apex, longueur du spicule gauche 240-250 $\mu$; longueur du spicule droit 300-315 $\mu$, longueur du gubernaculum $65 \mu$, longueur de la queue $350 \mu$.

Femelle: Longueur $31,5 \mathrm{~mm}$, largeur $600 \mu$, deirides, anneau nerveux et pore excréteur situés respectivement à $220 \mu, 300 \mu$ et $425 \mu$, bosse cuticulaire large de $250 \mu$, située à $1,8 \mathrm{~mm}$; deirides, anneau nerveux et pore excréteur situés respectivement à $220 \mu, 300 \mu$ et $425 \mu$; longueurs respectives du pharynx, de l'œsophage musculaire et de l'œsophage glandulaire $315 \mu$ et $915 \mu$, vulve située à $20,7 \mathrm{~mm}$ de l'apex, dimensions des œufs $50 \times 30 \mu$, longueur de la queue $60 \mu$.

Ces dimensions sont plus importantes que celles relevées sur les Spirura parasites de Tupaia. Cependant, les rapports des différents organes entre eux et notamment la position de la vulve au tiers postérieur du corps sont comparables. Nous pensons donc que le Spirura parasite de Nycticebus coucang à Bornéo ( ${ }^{\circ}$ d'enregistrement D.M.E. 4197), appartient à la même espèce que ceux parasites des Tupaia de Malaisie occidentale ( $\mathrm{n}^{\circ}$ d'enregistrement M.N.H.N. KL 122). 


\section{MoRPhOlOGIE LARVAIRE.}

Des larves du premier stade et des larves infestantes sont obtenues après un développement de 6 jours et de 43 jours chez des Blattes Blatella germanica infestées avec les œufs embryonnés du parasite récolté chez le Tupaia glis, KL 124 ; les Insectes sont maintenus à une température de $25^{\circ} \mathrm{C}$.

Des larves du troisième et du quatrième stade résultant d'une infestation spontanée sont récoltées chez le Tupaia glis KL 122.

Premier stade (fig. $3 \mathrm{~A}$ ) : La tête porte un fort crochet cuticulaire sur la surface gauche et plusieurs rangées d'épines. L'extrémité caudale pointue est ornée d'une couronne de fines aspérités (fig. $3 a$ ). L'œsophage est réduit dans sa partie antérieure à un simple axe cuticulaire et présente dans sa partie moyenne et sa partie postérieure deux massifs cellulaires. Cinq noyaux sont visibles dans l'ébauche intestinale, l'ébauche du rectum présente les deux noyaux des cellules prérectales et les noyaux des cellules $R_{2}, R_{3}, R_{4}$ disposés en triangle. La cellule $R_{1}$ plus volumineuse, située en avant du rectum, est divisée.

Les dimensions de cette larve sont: longueur $244 \mu$; largeur $15 \mu$; longueur de l'œsophage $134 \mu$; longueur de l'intestin $65 \mu$; longueur de la queue $50 \mu$.

Forme infestante (fig. $3 B$ ): Ses structures céphaliques (fig. $3 C-D$ et $E$ ) sont identiques à celles du Spirura adulte: le cadre buccal, comprimé latéralement au niveau des amphides, s'évase dorsalement et ventralement en une collerette cuticulaire. Les papilles céphaliques sont volumineuses. Le pharynx est aplati latéralement. Il existe dans la région œsophagienne une ébauche de la bosse cuticulaire (fig. $3 \mathrm{H}$ ) du Spirura adulte. Deux ailes latérales sont présentes (fig. $3 G$ ). L'extrémité caudale est formée d'un bourgeon de sept à huit pointes (fig. $3 \mathrm{~K}$ ).

Les dimensions de cette larve sont : longueur $4160 \mu$ à $4200 \mu$; largeur $110 \mu$; deirides, anneau nerveux et pore excréteur situés respectivement à $110 \mu, 165-185 \mu$ et $200 \mu$ de l'apex, bosse cuticulaire située à $900 \mu$ de l'apex ; longueur du pharynx 37-40 $\mu$; longueurs respectives de l'œsophage musculaire et de l'œsophage glandulaire 200 à $210 \mu$ et $2080 \mu$; longueur de l'intestin $1600 \mu$; ébauche génitale longue de $410 \mu$, située dans la dernière partie du corps ; longueur de la queue 120 à $130 \mu$.

Troisième stade larvaire chez le Tupaia (fig. 3I): Cette larve ne diffère de la larve infestante que par la croissance générale du corps et par l'allongement de l'ébauche génitale.

Ses dimensions sont : longueur $5260 \mu$; largeur $130 \mu$; deirides, anneau nerveux et pore excréteur situés respectivement à $115 \mu, 170 \mu$ et $220 \mu$ de l'apex; bosse cuticulaire œsophagienne située à $850 \mu$ de l'apex ; longueurs respectives du pharynx, de l'œsophage musculaire et de l'œsophage glandulaire $40 \mu, 210 \mu$ et $2400 \mu$; vulve située à $1200 \mu$ de l'extrémité caudale; longueur de l'ébauche génitale $1200 \mu$; longueur de la queue $155 \mu$.

Quatrième stade larvaire (fig. $3 \mathrm{~J}$ ): Cette larve femelle diffère de la larve du $3^{\circ}$ stade par la perte du bourgeon de denticules caudaux (fig. $2 M$ ) et par le développement de l'ovéjecteur (fig. $3 P$ ) et des deux utérus. 


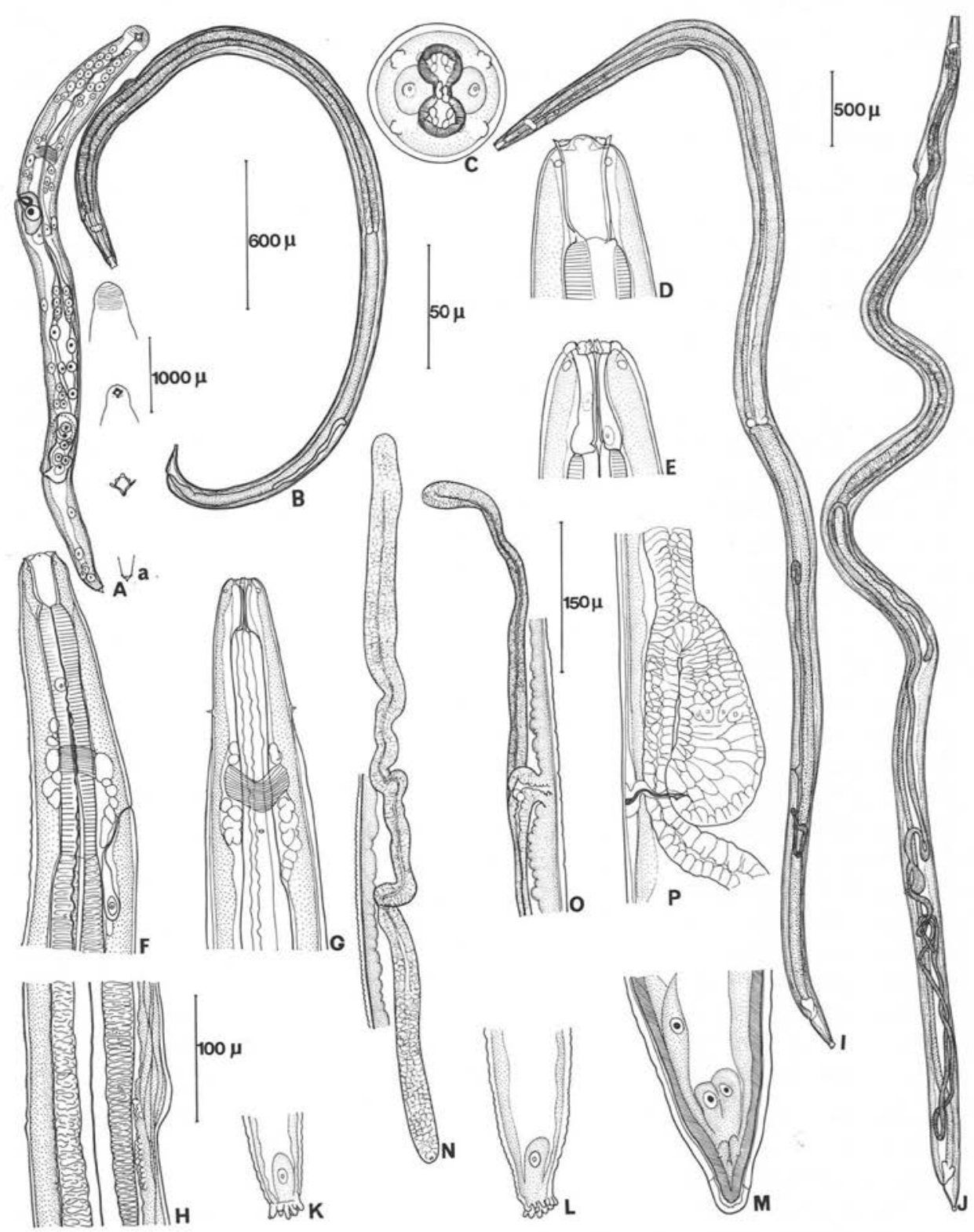

Fig. 3. - Spirura malayensis n. sp. Morphologie larvaire. A: larve du $1^{\text {er }}$ stade après six jours de développement à $25{ }^{\circ} \mathrm{C}$ chez Blatella germanica: a) détail de l'extrémité apicale de la larve sur les faces droite et gauche, détail du crochet cuticulaire, détail de la pointe caudale; B : larve du $3^{\circ}$ stade chez l'Insecte après 43 jours de développement à $25{ }^{\circ} \mathrm{C} ; \mathrm{C}, \mathrm{D}, \mathrm{E}$ : tête de la larve infestante en vues apicale, latérale et dorsale ; F et $\mathrm{G}$ : extrémité antérieure du corps de cette larve en vue latérale et ventrale; $\mathrm{H}$ : ébauche de bosse cuticulaire œsophagienne; I: larve $q$ du $3^{\text {e }}$ stade chez le Tupaia. J : larve $q$ du $4^{\text {e }}$ stade chez le Tupaia; K, L: bourgeons caudaux des larves du $3^{\mathrm{e}}$ stade chez l'Insecte et chez le Tupaia; M : extrémité caudale de la larve du $4^{e}$ stade; N, O, P: développement de l'ébauche génitale de la larve du $3^{\text {e }}$ stade chez l'Insecte, de la larve du $3^{\circ}$ stade chez le Tupaia et de la larve du $4^{\circ}$ stade chez le Tupaia.

A, C, D, E, K, L, M : éch. $50 \mu ;$ F, G, H, N, O : éch. $100 \mu$; B, I : éch. $600 \mu$; $\mathrm{J}$ : éch. $500 \mu ; \mathrm{P}$ : éch. $150 \mu$. 
Les dimensions de cette larve sont: longueur $9 \mathrm{~mm}$; largeur $210 \mu$; deirides, anneau nerveux et pore excréteur situés respectivement à 125, 200 et $250 \mu$ de l'apex ; longueurs respectives du pharynx, de l'œsophage musculaire et de l'œsophage glandulaire $40 \mu, 300 \mu$ et $3650 \mu$; vulve située à $2200 \mu$ de la pointe caudale, longueur de la queue $200 \mu$.

Spirura aurangabadensis (Ali et Lovekar, 1966).

Hôte: Nycticebus coucang (Boddaert).

Localité : Ulu Gombak, Forest reserve, Gombak, Selangor, Malaisie occidentale ; date de récolte : 18-12-74.

Matériel étudié : $5 \sigma^{*}+1 \uparrow, \mathrm{n}^{\circ}$ d'enregistrement M.N.H.N. P X 92.

Hôte : Tupaia glis.

Localité : Ulu Gombak, Forest reserve, Gombak, Selangor, Malaisie occidentale ; date de récolte : 2-1-74.

Matériel étudié : 1 entière immature, extrémité antérieure d'une femelle plus âgée ; $\mathrm{n}^{\circ}$ d'enregistrement M.N.H.N. KL 161.

\section{MORPHOLOGIE.}

La morphologie de ce Spirura est tout à fait remarquable par ses structures céphaliques, cuticulaires et génitales.

- Structures céphaliques (fig. 4A-B-C) : le pharynx est aplati latéralement. Le cadre buccal, issu de la paroi cuticulaire du pharynx, recouvre en partie l'extrémité céphalique et forme une collerette allongée dorso-ventralement, prolongée par une pointe sur chacune des faces dorsale et ventrale et échancrée au niveau des amphides. Ce cadre buccal porte six fortes dents non dédoublées, mais allongées et terminées en pointe.

L'aspect des terminaisons sensorielles est également très particulier. Les quatre papilles labiales qui sont petites chez les autres Spirura sont ici importantes et débordent le cadre buccal; par contre, les papilles céphaliques ne sont pas volumineuses mais très réduites.

- Ornementation cuticulaire: la paroi du corps est épaisse (fig. $4 \mathrm{E}$ ). Elle est ornée de stries cuticulaires profondes, elles-mêmes séparées par des stries beaucoup plus fines (fig. $4 \mathrm{~J}$ ). La bosse cuticulaire ventrale des Spirura est ici très développée (fig. $4 F$ et $G$ ) et maintient fortement le Nématode à la muqueuse œsophagienne. La paroi postérieure de ce repli cuticulaire s'incurve en une ventouse. A son niveau, le corps du Nématode est replié sur lui-même (fig. $4 \mathrm{D}$ ). La vulve $(f i g .4 \mathrm{~J}$ ) est entourée d'une plage de granulations cuticulaires, étirée longitudinalement. L'extrémité caudale de la femelle ne porte aucun bourgeon caudal.

- Structures génitales du mâle (fig. $4 \mathrm{H}$ ) : la particularité de la bourse caudale du mâle réside dans l'absence d'ornementation cuticulaire et dans la réduction du nombre de papilles cloacales. Celles-ci sont pédonculées et au nombre de quatre paires 


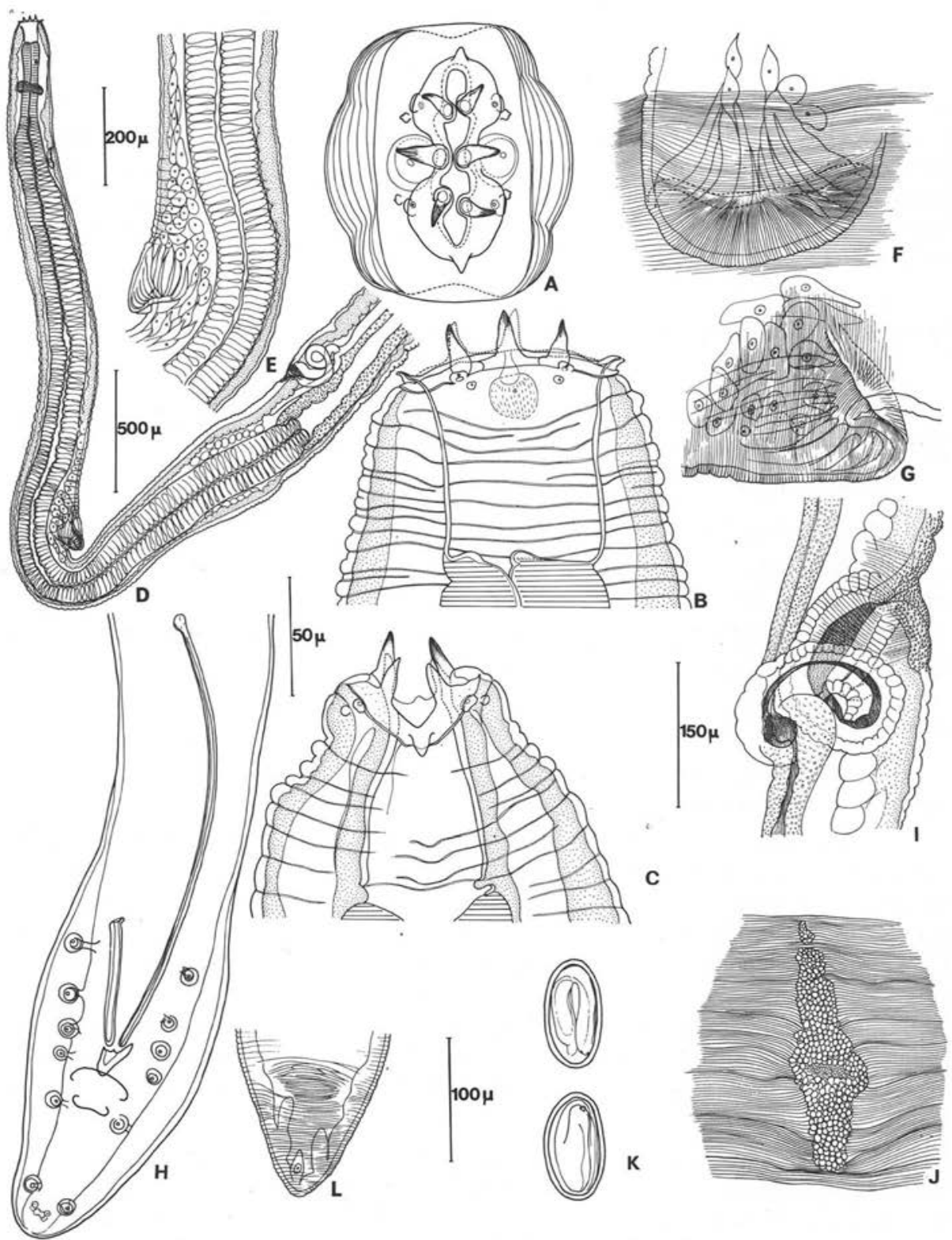

Fig. 4. - Spirura aurangabadensis (Ali et Lovekar, 1966)

A, B et C: tête représentée en vues apicale, latérale et dorsale, noter l'hypertélie des dents buccales, le développement de la collerette céphalique et la réduction des papilles céphaliques ; D : extrémité antérieure du corps, vue latérale ; E : développement des cellules souscuticulaires au niveau de la bosse cuticulaire œsophagienne ; F, G: bosse cuticulaire œsophagienne en vues ventrale et latérale ; $\mathrm{H}$ : bourse caudale mâle, vue ventrale ; I : dśtail de l'ornementation cuticulaire de la vulve et de l'ovéjecteur enroulé; J : détail de l'ornementation cuticulaire de la vulve en vue ventrale; K: œufs; L: extrémité caudale d'une jeune femelle.

A, B, C, K : éch. $50 \mu$; D : éch. $500 \mu$; E, H: éch. $200 \mu ; \mathrm{F}, \mathrm{G}, \mathrm{J}, \mathrm{L}$ : éch. $100 \mu$; $\mathrm{I}$ : éch. $150 \mu$. 
dans la région précloacale, mais dans la région postcloacale il n'existe plus que deux paires de papilles pédonculées et une paire de papilles très petites et sessiles. Les spicules sont très inégaux, le gauche étant environ quatre fois plus long que le droit.

- Ovéjecteur (fig. $4 \mathrm{I}$ ) : le vagin a une paroi épaisse et musculaire. Il se replie sur lui-même en une boucle avant de se prolonger en une branche utérine antérieure et une branche utérine postérieure.

\section{Dimensions des spécimens parasites du Nycticèbe ( $P X$ 92)}

Mâles: Leurs longueurs varient de 14 à 15,7 mm. Les dimensions du plus grand spécimen sont : largeur $500 \mu$, bosse cuticulaire œsophagienne située à $4 \mathrm{~mm}$ de l'apex, deirides, anneau nerveux et pore excréteur situés respectivement à $280 \mu, 470 \mu$ et $880 \mu$ de l'apex ; profondeur du pharynx $140 \mu$, largeur du pharynx $60 \mu$, longueurs respectives de l'œsophage musculaire et de l'œsophage glandulaire $510 \mu$ et $5800 \mu$, coude testiculaire situé à $7 \mathrm{~mm}$ de l'apex, longueur du spicule droit $260 \mu$, longueur du spicule gauche $930 \mu$, longueur du gubernaculum $90 \mu$, longueur de la queue $360 \mu$.

Femelle: Longueur 20,4 mm, largeur $700 \mu$, bosse cuticulaire œsophagienne située à 4,9 $\mathrm{mm}$ de l'apex, deirides, anneau nerveux et pore excréteur situés à $310 \mu$, $510 \mu$ et $1040 \mu$ de l'apex, profondeur du pharynx $180 \mu$, largeur $110 \mu$, longueurs respectives de l'œsophage musculaire et de l'œsophage glandulaire $460 \mu$ et $9400 \mu$, vulve située à $9400 \mu$ de l'apex, dimensions des œufs $46 \times 25 \mu$, longueur de la queue $300 \mu$.

\section{Dimensions des spécimens parasites du Tupaia (KL 161)}

La morphologie céphalique, cuticulaire et génitale de ces Spirura est tout à fait identique à celle des Spirura parasites du Nycticèbe ( ${ }^{\circ}$ PX 92).

Femelle: Les dimensions de cette femelle immature sont: longueur $7 \mathrm{~mm}$; largeur $330 \mu$; bosse cuticulaire œsophagienne située à $2100 \mu$ de l'apex ; deirides, anneau nerveux et pore excréteur situés respectivement à $155 \mu, 240 \mu$ et $400 \mu$ de l'apex ; profondeur du pharynx $85 \mu$; largeur du pharynx $55 \mu$, longueurs respectives de l'œsophage musculaire et de l'œsophage glandulaire $340 \mu$ et $3100 \mu$; vulve située à $3400 \mu$ de l'apex; longueur de la queue $125 \mu$.

Extrémité antérieure d'une seconde femelle: Ses dimensions sont: deirides, anneau nerveux et pore excréteur situés à $170 \mu, 280 \mu$ et $520 \mu$ de l'apex ; profondeur du pharynx $87 \mu$; largeur du pharynx $66 \mu$; longueur de la collerette céphalique $92 \mu$; largeur du rebord de la collerette $15 \mu$; longueur des pointes buccales $23 \mu$; longueurs respectives des œsophages musculaire et glandulaire $280 \mu$ et $3200 \mu$; bosse cuticulaire œsophagienne située à $2200 \mu$ de l'apex et mesurant $150 \mu$ de large et $110 \mu$ de haut ; vulve située à $3600 \mu$ de l'apex ; longueur de l'ovéjecteur enroulé sur lui-même jusqu'à la bifurcation des deux utérus $425 \mu$. 


\section{II. - Évolution et répartition des espèces du genre Spirura}

Les espèces $S$. malayensis n. sp. et $S$. aurangabadensis (Ali et Lovekar, 1966) appartiennent toutes deux au genre Spirura Blanchard, 1849 (1) (= Travassospirura Monnig, 1938 = Spiruracerca Erickson, 1938 = Taphozoia Ali et Lovekar, 1966) Chabaud, 1975, car ces Spirurides présentent en commun les caractères morphologiques suivants: pharynx aplati latéralement, structures cuticulaires buccales non recouvertes par des néoformations labiales, présence d'une bosse cuticulaire simple ou double dans la région œsophagienne.

La réalisation des cycles biologiques de Spirura rytipleurites rytipleurites par Stefanski, 1934, S. rytipleurites seurati par Chabaud 1954, S. talpae par Chabaud et Mahon, 1958, S. guianensis par Quentin, 1973 et S. malayensis n. sp. et l'étude de la morphogénèse des structures céphaliques du troisième stade larvaire à l'adulte, mettent en évidence deux tendances évolutives différentes chez ces Spirurides. Ces tendances permettent de répartir les espèces, au sein du genre Spirura, selon deux lignées (fig. 5).

Dans une première lignée, la collerette cuticulaire du cadre buccal est arrondie dans les régions dorsale et ventrale. Cette collerette est identique chez la larve et chez l'adulte, ou ne subit au cours de la morphogénèse larvaire que de légères échancrures au niveau des papilles labiales. Les dents buccales sont peu développées.

Nous considérons que ces structures sont les plus primitives car elles rappellent celles du genre Paraspirura Sandground, 1936 parasite d'un Reptile d'Afrique, dont la collerette céphalique est arrondie sur les faces dorsale et ventrale et dont le cadre buccal ne comporte pas de dents (cf. fig. 5).

Cette lignée est la plus importante car elle rassemble la majorité des espèces. Celles-ci restent très proches morphologiquement car leurs structures céphaliques restent stables. Elles peuvent cependant se distinguer les unes les autres par des structures génitales mâles de plus en plus spécialisées.

Cinq groupes peuvent ainsi être individualisés selon le degré de différenciation de ces structures (2); chacun d'eux correspondant à un spectre d'hôtes particulier.

- Le premier groupe rassemble les espèces les plus primitives. Les rebords ventraux et dorsaux de la collerette cuticulaire céphalique sont parfaitement arrondis. La bosse cuticulaire ventrale est quelquefois dédoublée. Une touffe relique d'épines caudales peut subsister chez les adultes.

La bourse caudale ne présente pas de différenciations cuticulaires localisées, les spicules sont de petite taille et dépassent rarement $350 \mu$. Il n'existe que quatre paires de papilles précloacales.

(1) Keahey et Trapp, 1969 ont signalé chez des Oiseaux rheiformes l'espèce Spirura zschokkei Railliet et Henry, 1911. Cette espèce est en réalité un Habronème et appartient au genre Odontospirura Wehr, 1933, ainsi que l'a mis en évidence Chabaud, 1958.

(2) Nous rangeons dans la première lignée l'espèce $S$. portesiana Campana et Chabaud, 1950 parasite de Xerus en Afrique, sans pouvoir toutefois préciser sa place zoologique exacte car le mâle de cette espèce reste encore inconnu. 

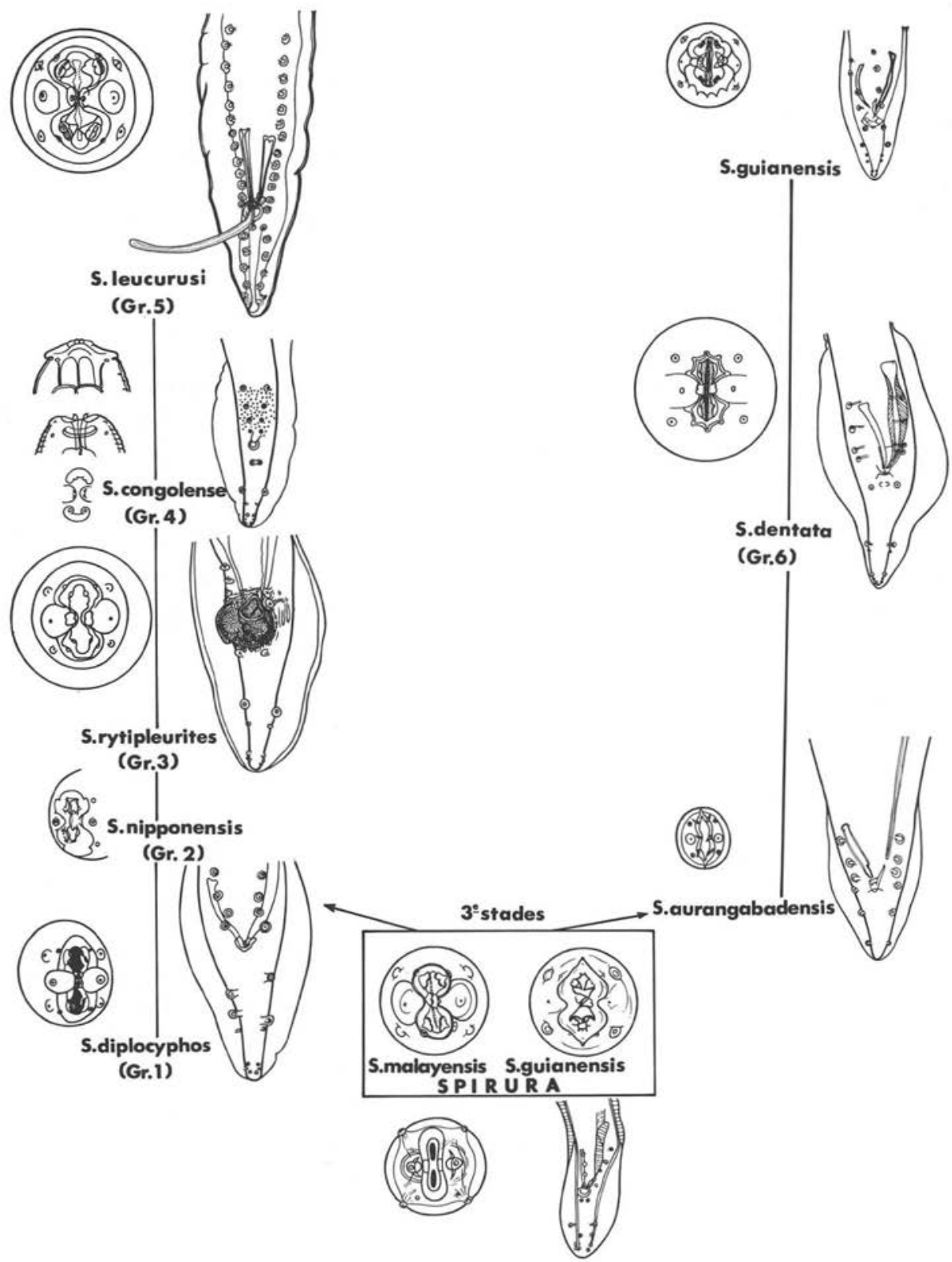

PARASPIRURA

FIg. 5. - Schéma représentant l'évolution hypothétique des Nématodes du genre Spirura Dans une première lignée, les structures céphaliques restent stables, cinq groupes (Gr. 1, Gr. 2, Gr. 3, Gr. 4 et Gr. 5) peuvent être individualisés selon le degré de différenciation des structures génitales mâles.

Dans une seconde lignée ( $\mathrm{Gr}$. 6), le rebord de la collerette cuticulaire céphalique n'est plus arrondi mais se prolonge par une pointe dorsale et une pointe ventrale. Cette structure initiale se différencie ensuite par une ornementation plus complexe de la collerette cuticulaire céphalique et par l'hypertélie des dents buccales. 
Ce groupe compte actuellement trois espèces: gache.

- $S$. diplocyphos Chabaud, Brygoo et Petter, 1965 parasite de Lémurien mal-

- S. spinicaudata Chabaud, Brygoo et Petter, 1965 parasite de Lémurien d'Afrique. Prod'Hon, 1967 en a précisé la morphologie.

- S. rothschildi Seurat, 1915, parasite de Macroscelides d'Afrique du Nord.

Nous rattachons à ce groupe l'espèce Spirura malayensis n. sp., parasite des Tupaia et du Nycticèbe en Malaisie. Elle est en effet très proche de $S$. diplocyphos avec laquelle elle présente notamment une bosse cuticulaire œsophagienne double.

Ces deux espèces diffèrent cependant par la longueur relative des spicules et l'ornementation caudale de la femelle.

- Chez $S$. diplocyphos, le spicule gauche trapu est plus grand que le spicule droit alors que chez $S$. malayensis le spicule droit, plus fin, est égal ou plus allongé que le spicule gauche. Le bourgeon de dents caudales visible chez les femelles de $S$. diplocyphos disparaît chez $S$. malayensis dès le quatrième stade larvaire.

- Le deuxième groupe rassemble des espèces dont le cadre buccal est devenu légèrement échancré au niveau des papilles labiales. Ces espèces ne semblent pas présenter de différenciations cuticulaires locales au niveau du cloaque chez le mâle et de la vulve chez la femelle. Les spicules sont courts chez la plupart des espèces. Ce sont : Japon.

- S. nipponensis Ohbayashi, Masegi et Kubota, 1972 parasite de taupes au

- $S$. diana Ureche et Ureche, 1968 parasite du Furet en Europe.

- S. moldavica Andreiko, 1969 et S. talpae (Gmelin, 1790) parasites de taupes en Europe. Cette dernière espèce est également signalée chez le Rattus.

- Le troisième groupe réunit des espèces dont le cadre buccal est aussi échancré au niveau des papilles labiales, mais dont la cuticule est pourvue de différenciations localisées au niveau de la vulve chez la femelle et du cloaque chez le mâle. Les spicules sont toujours de grande taille et atteignent $600 \mu$. Ce sont :

- S. rytipleurites rytipleurites (Deslongchamps, 1824) $(=$ Filaria gastrophila Mueller, 1894) parasite du Chat, du Renard et du Rattus en Europe.

- $S$. rytipleurites seurati Chabaud, 1954 parasite d'Erinacéidés et de Carnivores Viverridés Mustelidés et Canidés d'Afrique du Nord.

- Le quatrième groupe réunit des espèces dont le nombre et la disposition des papilles cloacales restent d'un type primitif, mais dont les spicules sont devenus très inégaux par suite du développement considérable du spicule droit. Ce sont:

- S. narayani Mirza et Basir, 1938 et $S$. congolense Vuylsteke, 1956 parasites de Mangoustes aux Indes et en Afrique.

- Le cinquième groupe de cette lignée a été clairement défini par Babero, 1973. Les espèces qu'il rassemble sont caractérisées par une augmentation du nombre de papilles précloacales pouvant atteindre dix paires au moins de papilles et par des 
spicules très inégaux. Les dents buccales coniques sont bien individualisées. Toutes les espèces sont parasites de Rongeurs holarctiques Sciuridés et Zapodidés. Ce sont :

- S. michiganensis Sandground, 1935 et $S$. petrovi Gubanov, 1964 parasite d'Eutamias, S. zapi (Erickson, 1938) parasite de Zapus, S. infundiliformis Mc Leod, 1933 parasite de Citellus, S. leucurusi Babero, 1973 parasite d'Ammospermophilus.

- L'évolution de la seconde lignée de Spirura peut être retracée en suivant l'ontogénèse céphalique de $S$. guianensis. Chez cette espèce très différenciée, les structures apicales de la larve infestante, diffèrent, en effet, profondément de celles du quatrième stade et de l'adulte correspondant, mais rappellent éroitement celles de l'adulte de Spirura aurangabadensis.

Dans cette lignée le rebord de la collerette cuticulaire céphalique n'est plus arrondi, mais se prolonge par une pointe dorsale et une pointe ventrale. Cette structure initiale acquiert ensuite une ornementation plus complexe au niveau de la collerette cuticulaire céphalique et une hypertélie des dents buccales.

Trois espèces appartiennent actuellement à cette lignée. Nous les rassemblons dans un sixième groupe. Ce sont:

- S. aurangabadensis (Ali et Lovekar, 1966), parasite d'un Microchiroptère Taphozous kacchensis aux Indes. Nous identifions à cette espèce les spécimens récoltés chez le Nycticèbe (PX 92) et le Tupaia (KL 161) de Malaisie occidentale, car ils présentent en commun avec $S$. aurangabadensis les caractères suivants : cadre buccal prolongé par une pointe dorsale et une pointe ventrale, dents buccales allongées, bosse cuticulaire très développée située dans la première partie du corps en arrière de la jonction œsophage-intestin, paroi de l'ovéjecteur épaissie et courbée, papilles postcloacales réduites en nombre, bourse caudale sans ornementation cuticulaire, spicules très inégaux.

L'ornementation cuticulaire plus accentuée et les dimensions des spicules, plus importantes chez les spécimens parasites du Nycticèbe $(930 \mu$ et $260 \mu)$ que chez ceux parasites du Taphozous $(490 \mu$ et $160 \mu)$, sont tout à fait compatibles avec la taille deux ou trois fois plus importante des Spirura parasites du Nycticèbe et ne justifient pas, à notre avis, la création d'une espèce nouvelle.

- S. dentata (Mönnig, 1938), parasite d'une Mangouste en Afrique. En plus des pointes médianes, les rebords cuticulaires céphaliques dorsaux et ventraux s'ornent chacun de quatre pointes latérales.

- S. guianensis (Ortlepp, 1924) (= S. tamarini Cosgrove, Nelson Jones, 1963), Thatcher et Porter, 1968, parasite de Primates et de Marsupiaux néotropicaux. Chez cette espèce, seul le rebord cuticulaire ventral porte cinq pointes, les dents buccales sont bifides.

En conclusion, l'évolution du genre Spirura paraît s'être réalisée selon deux lignées principales, dont les formes ancestrales peuvent être représentées par les Spirura de Lémuriens malgaches et africains.

Ce genre semble donc s'être principalement diversifié, dès l'origine, dans l'Ancien Monde, chez des hôtes (Lémuriens, Macroscélidés, Talpidés, Erinacéidés, Mustélidés, 
Viverridés, Canidés, Rongeurs Sciuridés) qui sont tous primitifs et dont les époques d'apparitions paléontologiques se sont échelonnées au cours du Tertiaire.

L'adaptation successive à ces hôtes différents aurait entraîné une différenciation croissante de caractères archaïques (allongement des spicules, accroissement du nombre de papilles cloacales, hypertélie du cadre et des dents buccales).

L'existence de formes plus différenciées ( $S$. guianensis), en Amérique du Sud, résulterait de leur isolement géographique chez des hôtes très anciens (Primates Platyrhyniens).

La présence de cette espèce chez des Marsupiaux néotropicaux serait due à une infestation secondaire, écologique. L'étude précédente sur l'évolution générale du genre Spirura montre, en effet, que les formes les plus primitives sont parasites de Lémuriens dont l'apparition paléontologique est postérieure à celle des Marsupiaux.

\section{REMERCIEMENTS}

Ce travail a été entrepris à la Division d'Ecologie médicale de l'Institut de Recherche médicale de Kuala Lumpur (Malaisie). Nous exprimons notre reconnaissance à son directeur, M. Lim Boo Liat, et à son équipe pour l'aide qu'ils nous ont apportée dans la récolte des parasites.

Nous remercions également M. G. Stanley de Silva (Office of the Chief Game Warden) pour l'accueil qu'il nous a réservé à la station biologique de Sepilok Sandakan (Sabah).

\section{Bibliographie}

Ali (S. M.) et Lovekar (C. D.), 1966. - On a new Spirurid Taphozia aurangabadensis n. g. n. sp. From a micro-bat Taphozous kacchensis. Ind. J. Helmint., 18, 68-73 (Seminar Suppl.).

ANDREIKo (O. F.), 1969. - Nématodes d'Insectivores de la région située entre les rivières Prut et Dnestr - in Spasski A.A. (Ed.). Parasites de Vertébrés. Kishinev Izdat. Kartya Moldovenyaske, 146-155.

Babero (B. B.), 1973. - Nematodes of Nevada ground squirrels, with description of two new species. Trans. Amer. Micros. Soc., 92, 265-272.

Blanchard (E.), 1848-1849. - Recherches sur l'organisation de vers. Ann. Sci. Nat. Zool., $10,321-364 ; 11,106-202 ; 12,267-276$.

Chabaud (A. G.), 1954. - Sur le cycle évolutif des Spirurides et de Nématodes ayant une biologie comparable. Valeur systématique des caractères biologiques. Ann. Parasit. hum. comp., 29, 42-88, 206-249, 358-425.

Chabaud (A.-G.), 1958. - Essai de classification des Nématodes Habronematinae. Ibid., 33 (4), 445-508.

Снаваud (A.-G.), 1975. - Keys to genera of the Order Spirurida. Part. 2. Spiruroidea, Habronematoidea and Acuarioidea. C.I.H. Keys to the Nematode Parasites of Vertebrates $\mathrm{n}^{\circ}$ 3. (Anderson, Chabaud et Willmott, éd.). St-Albans, Angleterre, 29-58. 
Chabaud (A.-G.), Brygoo (E. R.) et Petter (A. J.), 1965. - Les Nématodes parasites des Lémuriens malgaches. Ann. Parasit. hum. comp., 40, 181-214.

Chabaud (A.-G.) et Mahon (J.), 1958. - Cycle évolutif du Nématode Spirura talpae (Gmelin, 1790). C.R.S. Soc. Biol., 152, 474-476.

Cosgrove (G. E.), Nelson (B. M.) et Jones (A. W.), 1963. - Spirura tamarini sp. n. (Nematoda: Spiruridae) from an Amazonian Primate, Tamarinus nigricollis (Spix, 1923), J. Parasit., 49, 1010-1013.

Deslongchamps (E.-E.), 1824. - Filaire-Filaria. Encyclopédie méthodique, Paris, II, 391-397.

Erickson (A. B.), 1938. - Parasites of some Minnesota Cricetidae and Zapodidae, and a Host Catalogue of Helminth Parasites of Native American Mice. Amer. Midland. Nat., 20, 575-589.

Fibiger (J.) et Ditlevsen (H.), 1914. - Contributions to the biology and morphology of Spiroptera (Gongylonema) neoplastica n. sp. Mindeskr. J. Japetus Steenstrup., $25,1-28$.

Gmelin (J. F.), 1790. - Carolia Linné, - Systema Naturea - Vermes, 1, 3032.

Gubanov (N. M.), 1964. - Helminthofaune de Mammifères d'importance économique dans la République de Yakut (U.R.S.S.), Izdatelsvo «Nauka», Moscou, 164.

KeyHeY (K. K.) et Trapp (A. L.), 1969. - Diagnoses and classifications of diseases of exotic animals. J. Am. vet. med. Ass., 155, 1136-1140.

MAC LeOd (J. A.), 1933. - A parasitological survey of the genus Citellus in ManitobaCanadian J. Research., 9, 108-127.

Mirza (M. B.) et Basir (M. A.), 1938. - On a collection of Nematodes from Hyderabad Deccan (India), Zeitsch. f. Parasitenkunde, 10, 217-220.

MöNNIG (H. D.), 1938. - A new Spirurid Nematode from a Mongoose. Livre. Jub. Pr. Travassos, $333-335+1$ pl.

Mueller (A.), 1895. - Filaria gastrophila sp. n. in a cat. Veterin., London (806), 68, $4^{e}$ sér., 41, 107 (808), n. s. (484), 267-268.

Ohbayashi (M.), Masegi (T.) et Kubota (K.), 1972. - Some nematodes of the Japanese shrew mole, Urotrichus talpoides, Temminck, Japanese Journ. Vet. Research, 20, 111-115.

Ortlepp (R. J.), 1924. - On a collection of Helminths from Dutch Guina. J. Helm., 2, $15-40$.

Prod'Hon (J.), 1967. - Etude de trois Nématodes Spirurides d'Angola. Publ. Cult. Co. Diam. Ang. Lisboa, 71, 45-60.

Quentin (J. C.), 1973. - Présence de Spirura guianensis (Ortlepp, 1924) chez des Marsupiaux néotropicaux. Cycle évolutif. Ann. Parasit. hum. comp., 48, 117-133.

Raillet (A.) et Henry (A.), 1911. - Les Helminthes du Nandou. Bull. Soc. Nat. Acclim., $58,538-541$.

Seurat (L.-G.), 1915. - Expedition de M.M. Walter Rothschild, E. Hartert et C. Hilgert dans le Sud Algérien (mars-mai 1914). Nématodes parasites. Novitat. Zool., 22, $1-25$. 
SANDground (J. H.), 1935. - Spirura michiganensis n. sp. and Rictularia halli n. sp. two new parasitic nematodes from Eutamias striatus lysteri (Richardson). Trans. Am. Micr. Soc., 54, 155-166.

SANDGRound (J. H.), 1936. - Scientific results of an expedition to rain forest region in Eastern Africa. Bull. Mus. Comp. Zool., 79, 341-366.

StefansKY (W.), 1934. - Sur le développement et les caractères spécifiques de Spirura rytipleurites (Deslongchamps, 1824). Ann. Parasit. hum. comp., 12, 203-217.

Thatcher (V. E.) et Porter (J. A.), 1968. - Some helminths parasites of Panamanian Primates. Trans. Amer. Microsc. Soc., 87, 186-196.

URECHE (L.) et URECHE (A.), 1968. - Identificarea unei specii noi de nematod gastric din familia spiruridae. La viezure in tara noastra. Revta Zootech. Med. Vet. Buc., $18,55-60$.

VUYLSTEKE (C.), 1956. - Note sur quelques Nématodes parasites avec description de neuf espèces nouvelles. Rev. Zool. Bot. Afr., 53, 441-477.

WeHR (E. E.), 1933. - A new Nematode from the Rhea. Proc. U.S. Nat. Mus., 82, 1-5. 